\title{
Predicting Breast Cancer: A Comparative Analysis of Machine Learning Algorithms
}

\author{
Pulung Hendro Prastyo*, I GedeYudi Paramartha**, Michael S. Moses Pakpahan***, Igi Ardiyanto**** \\ Department of Electrical Engineering and Information Technology, Faculty of Engineering, Universitas Gadjah Mada \\ Jalan Grafika No. 2 Kampus UGM, Yogyakarta 55281, Indonesia. \\ Email: pulung.hendro@mail.ugm.ac.id*, gedeyudi@mail.ugm.ac.id**,msmpakpahan@mail.ugm.ac.id***, igi@ugm.ac.id****
}

\begin{abstract}
Breast cancer is the most common cancer among women (43.3 incidents per 100.000 women), with the highest mortality (14.3 incidents per 100.000 women). Early detection is critical for survival. Using machine learning approaches, the problem can be effectively classified, predicted, and analyzed. In this study, we compared eight machine learning algorithms: Gaussian Naïve Bayes (GNB), kNearest Neighbors (K-NN), Support Vector Machine(SVM), Random Forest (RF), AdaBoost, Gradient Boosting (GB), XGBoost, and Multi-Layer Perceptron (MLP). The experiment is conducted using Breast Cancer Wisconsin datasets, confusion matrix, and 5-folds cross-validation. Experimental results showed that XGBoost provides the best performance. XGBoost obtained accuracy $(97,19 \%)$, recall (96,75\%), precision (97,28\%), F1-score $(96,99 \%)$, and AUC $(99,61 \%)$. Our result showed that XGBoost is the most effective method to predict breast cancer in the Breast Cancer Wisconsin dataset.
\end{abstract}

Keywords: XGBoost, MachineLearning, Breast Cancer, Classification

\section{INTRODUCTION}

Breast cancer is the most common cancer among women, with 43.3 incidents per 100.000 women. Breast cancer has a relatively low fatality rate with other types of cancer. However, with a large number of incidents, it has the highest mortality rate of any cancer among women (12.9 per 100 000). Early detection is critical for survival. Approximately $70 \%$ of deaths from cancer occur in low- and middle-income countries. Limited resources with underdeveloped health systems making it difficult for the patient to get access to the medical professional. Developing early diagnosis programs based on early signs and symptoms can improve the patient survival rate. (World Health Organization. 2019).

With a growing dataset of breast cancer patients, it is more feasible that machine learning methods are implemented to provide a quick, automated, and deeper understanding of cancer healthcare (Maity, G., and Das, S. 2017). Detection requires accurate prediction, and available large datasets give us the opportunity for an accurate prediction. However, the problem lies in what method will provide us with the best result.

Previous research has compared various machine learning methods to predict breast cancer. Using the Wisconsin Breast Cancer dataset, a performance comparison of Support Vector Machines or SVM, Decision Tree (C4.5), Naïve Bayes, and k-Nearest Network or kNN were conducted by Asri, $\mathrm{H}$ et al. (Asri, H. et al. 2016). SVM reached the highest accuracy by 97,13\% and outperformed other algorithms. Bayrak, E. et al. (Bayrak, E. et al. 2019) compared SVM and Artificial Neural Network or ANN to predict breast cancer in early stages. The result showed that SVM has the best performance of 96,9957\%. Gbenga, D. et al. (Gbenga, D. et al. 2017) compared eight machine learning algorithms to predict breast cancer using WEKA data mining and machine learning simulation environment. Algorithms compared in this research are SVM, Radial Based Function, Simple Linear Logistic Regression Model, Naïve Bayes, kNN, AdaBoost, Fuzzy Unordered Role Induction algorithm, and Decision Tree (J48). Their experimental result indicated that SVM has the best performance (97.07\%).

Though previous research showed that SVM comparatively is the most accurate method, other comparative studies showed accurate methods. Amrane, M. et al. (Amrane, M. et al. 2018) compared two different classifiers: Naïve Bayes and $\mathrm{kNN}$ for breast cancer classification. They used cross-validation methods as a tool to evaluate accuracy. The result showed that K-NN gives better accuracy than Naïve Bayes $(97,51 \%)$. Sharma, S. et al. (Sharma, S. et al. 2018) compared Random Forest or RF, K-NN, and Naïve Bayes to predict breast cancer using The Wisconsin Breast Cancer dataset. The research concluded that KNN has the highest accuracy of $94,20 \%$. Liu, B. et al. (Liu, B. et al. 2018) compared several machine learning methods including SVM, AdaBoost, Decision Tree, and Random Forest or RF to predict the benign and malignant of breast cancer from a digitized image of a fine needle aspirate of a breast mass. Theresult showed that the random forest is the best method for prediction.

These results show that comparative research can be done on these methods. An objectively best method can 
be identified using a common dataset. There are other methods that have never been compared to predict breast cancer. Therefore, we have evaluated the performance of the following machine learning algorithms: Gaussian Naïve Bayes (GNB), K-Nearest Neighbors (K-NN), Support Vector Machine (SVM), Random Forest (RF), AdaBoost, Gradient Boosting (GB), XGBoost, and Multi-Layer Perceptron (MLP).

\section{MATERIALS AND METHODS}

\section{Dataset}

The Wisconsin Breast Cancer dataset from UCI Machine Learning Repository was used in this experiment. There are 30 numeric attributes of features in the dataset. Features are calculated from a digitized image of a fine needle aspirate (FNA) of a breast mass. They describe the characteristics of the cell nuclei present in the image. Dataset has 569 instances that divided into two classes: benign and malignant. Benign consists of 212 instances, and malignant consists of 357 instances (UCI, 2019).

\section{Experiment Method}

Design of the experiment is presented in Figure 1:

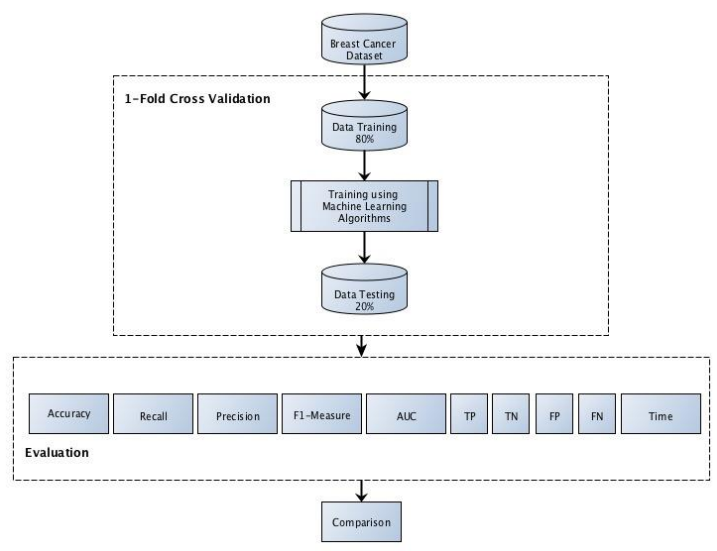

Figure 1. 1-Fold Cross Validation.

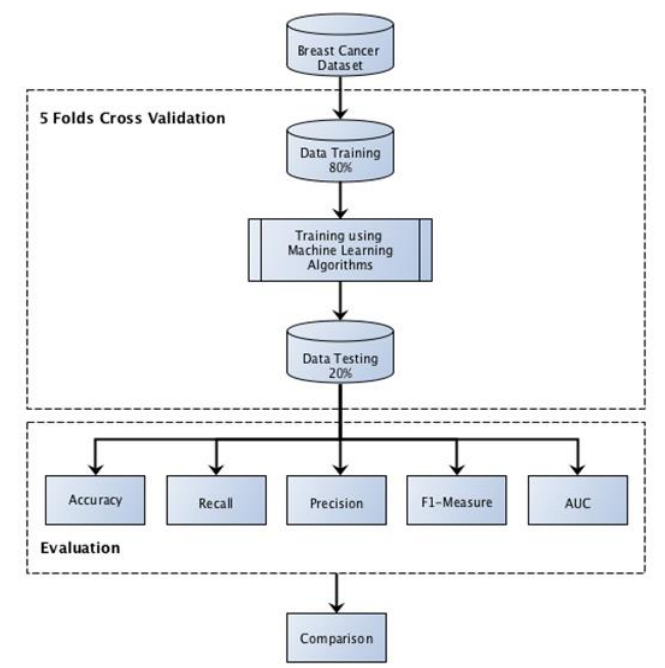

Figure 2. 5-Fold Cross Validation.
As we can see in Figure 1 and Figure 2, we used two ways to validate the performance of machine learning algorithms: (1) 1-Fold Cross-Validation and (2) 5-Folds Cross-Validation. Performance metrics that we used are accuracy, recall, precision, F1-Score, and Area Under Curve (AUC). In this experiment, we split the data into $80 \%$ data training and $20 \%$ data testing. Then, we train and test every algorithm for one iteration (1-fold crossvalidation). After that, we run five iterations (5-folds cross-validation) using different, randomized data training and data testing from the dataset. Finally, we average every performance metric. All processes of the experiment are performed with Python 3.7 on a Laptop with $2.5 \mathrm{GHz}$ Intel Core i5 and $16 \mathrm{~GB}$ RAM, running on macOS High Sierra.

\section{a. Peformance Metrics/Confusion Matrix}

First, the definition of true positive, true negative, false positive, and false negative are defined in Table 1.

Table 1. Definitions of True Positive, True Negative, False Positive, and False Negative.

\begin{tabular}{cccc} 
& & \multicolumn{2}{c}{ Predicted class } \\
\cline { 3 - 4 } Actual & & Class $=$ True & Class $=$ False \\
\cline { 2 - 4 } class & Class $=$ True & True positive & False Negative \\
& Class $=$ False & False Positive & True Negative \\
\cline { 2 - 4 }
\end{tabular}

Performance metrics that we used are described as follows:

1) Accuracy

Accuracy represents a degree of correctness in the training of the model. It is defined as the measurement of correct prediction compared to all predictions. The equation for accuracy is presented below:

Accuracy $=$

(True Positive+True Negative) $\overline{\text { (True Positive }+ \text { False Positive }+ \text { True Negative }+ \text { False Negative) }}$

2) Recall

Recall is a ratio to correctly determined positive instances to True Positive and False Negative. The equation for recall is presented below:

Recall $=\frac{\text { True Positive }}{(\text { True Positive }+ \text { False Negative })}$

3) Precision

Precision is a degree of correctness in determining the ratio between True Positives and all positive prediction. The equation for precision is presented below:

Precision $=\frac{\text { True Positive }}{(\text { True Positive }+ \text { False Positive })}$

4) F1-Score/F-Measure

F1-Score is a weighted average of Precision and Recall. The equation for F1-Scores is presented below:

F1-Score $=\frac{2 *(\text { Precision } * \text { Recall })}{(\text { Precision }+ \text { Recall })}$ 


\section{b. $A U C$}

AUC measures accuracy by measuring the percentage of area under ROC curve. Wider the area under the ROC curve means that an algorithm has a better accuracy (Derisma, et al. 2018)

\section{c. K-Fold Cross Validation}

Cross-validation is a statistical technique used to check and evaluate learning algorithms or models. Crossvalidation splits data randomly into a learning set to train and a testing set to evaluate the model. K-fold cross- validation is the basic form, one of the $\mathrm{k}$ partitions it is used as a validation set. We used k-fold cross-validation to validate performance metrics, has it represented the entire dataset (Suyanto. 2018).

\section{Machine Learning Algorithms}

In this section, we will describe every algorithm that we used in this experiment. Table 2 describes each basic algorithm concept, especially in classifying problem:

Table 2. Basic Concept of Each Algorithm.

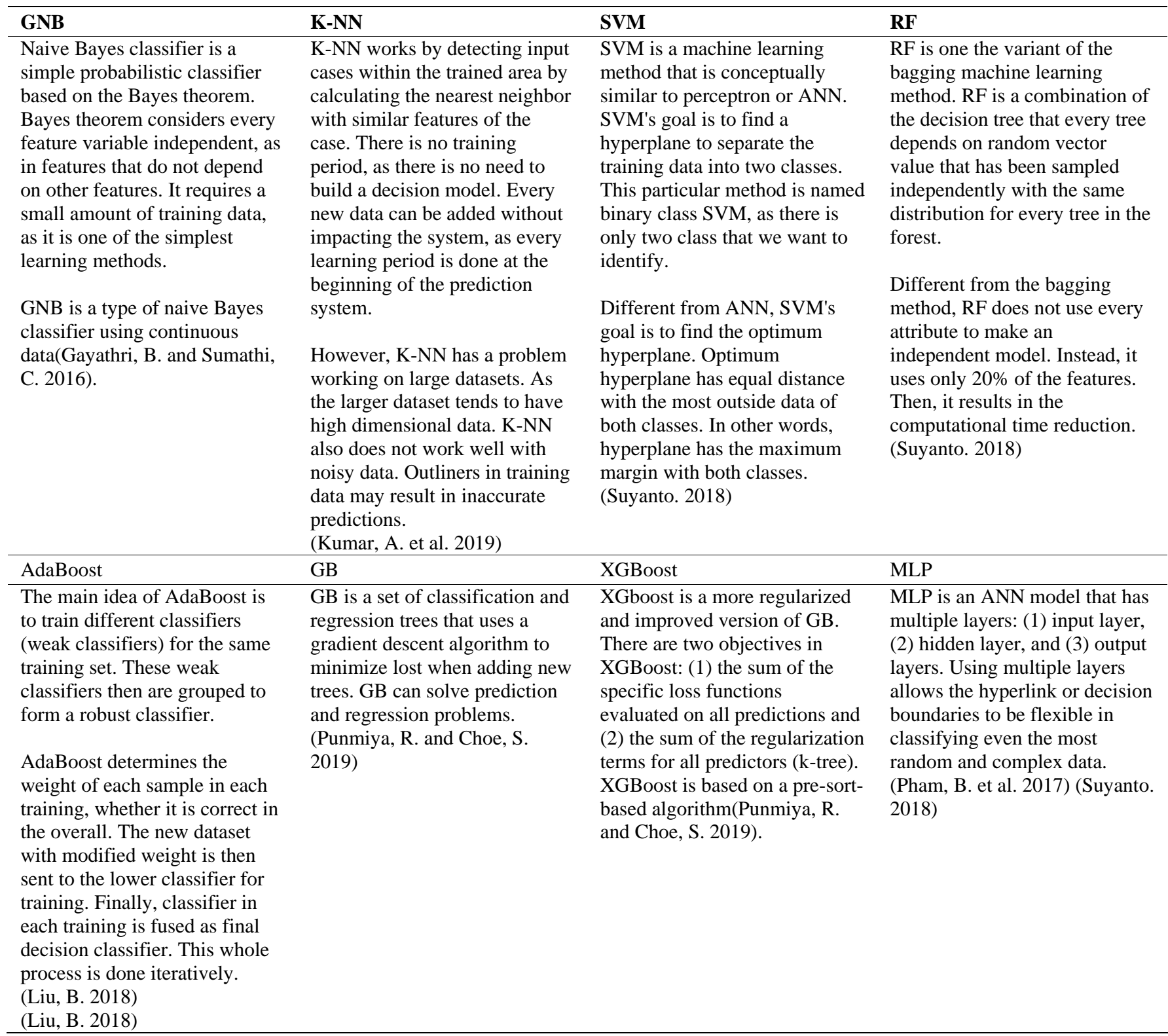




\section{RESULTS AND DISCUSSION}

\section{Comparison Amongthe Algorithms with 1-Fold Cross Validation}

At first, we compared the algorithms with 1-fold cross validation method. The result are shown in Table 3:

As we can see in Table 1, excluding computing time, XGBoost outperforms other algorithms. In context, XGBoost correctly classifies two more cases than the second-best algorithm, KNN. Even though it is lower than GNB, XGBoost also has better computational time than other algorithms, because it takes 0.08 seconds to process. GNB performed better, only requires 0.008 seconds to process. Interestingly, the SVM method, one of the best algorithms in previous studies, has the longest computational time and is one of the lowest performance metric results as well.

Table 3. Performance Metrics with 1-Fold Cross Validation.

\begin{tabular}{|c|c|c|c|c|c|c|c|c|c|}
\hline Algorithm & Recall & Precision & F1-Score & Accuracy & $\mathbf{T P}$ & $\mathbf{T N}$ & FP & $\mathbf{F N}$ & Time \\
\hline GNB & $94,03 \%$ & $94,03 \%$ & $94,03 \%$ & $92,98 \%$ & 63 & 43 & 4 & 4 & $0,008 \mathrm{~s}$ \\
\hline $\mathrm{kNN}$ & $98,51 \%$ & $95,65 \%$ & $97,06 \%$ & $96,49 \%$ & 66 & 44 & 3 & 1 & $0,12 \mathrm{~s}$ \\
\hline SVM & $94,03 \%$ & $98,43 \%$ & $96,18 \%$ & $95,61 \%$ & 63 & 46 & 1 & 4 & $1,57 \mathrm{~s}$ \\
\hline $\mathrm{RF}$ & $94,03 \%$ & $98,44 \%$ & $96,18 \%$ & $95,61 \%$ & 63 & 46 & 1 & 4 & $0,23 \mathrm{~s}$ \\
\hline AdaBoost & $97,01 \%$ & $95,59 \%$ & $96,30 \%$ & $95,61 \%$ & 65 & 44 & 3 & 2 & $0,14 \mathrm{~s}$ \\
\hline GB & $97,01 \%$ & $97,01 \%$ & $97,01 \%$ & $96,49 \%$ & 65 & 45 & 2 & 2 & $0,23 \mathrm{~s}$ \\
\hline XGBoost & $98,51 \%$ & $98,51 \%$ & $98,51 \%$ & $98,25 \%$ & 66 & 46 & 1 & 1 & $0,08 s$ \\
\hline MLP & $95,52 \%$ & $94,12 \%$ & $94,81 \%$ & $93,86 \%$ & 64 & 43 & 4 & 3 & $0,91 \mathrm{~s}$ \\
\hline
\end{tabular}

\section{Comparison AmongtheAlgorithmswith5-fold Cross Validation Method}

We implemented5-fold cross validation method and average performance metric was taken. Result are shown in Table 4:

Similar to the previous result in Table 3, XGBoost has the best result compared to other algorithms.
Performance metrics of XGBoost are Recall 96,75\%, Precision 97,28\%, F1-Score 96,99\%, and Accuracy $97,19 \%$. XGBoost also has the highest AUC of $99,61 \%$. From the experiment, we can conclude that XGBoost is the most accurate algorithm to classify breast cancer using the Wisconsin Breast Cancer dataset.

Table 4. Performance Metrics with Cross Validation.

\begin{tabular}{lllll}
\hline Algorithm & Recall & Precision & F1-Score & Accuracy \\
\hline GBN & $93,30 \%$ & $94,77 \%$ & $93,82 \%$ & $94,21 \%$ \\
kNN & $90,49 \%$ & $92,55 \%$ & $91,26 \%$ & $91,93 \%$ \\
SVM & $94,33 \%$ & $95,01 \%$ & $94,60 \%$ & $94,91 \%$ \\
RF & $95,45 \%$ & $95,82 \%$ & $95,05 \%$ & $94,91 \%$ \\
AdaBoost & $95,34 \%$ & $96,21 \%$ & $95,71 \%$ & $95,96 \%$ \\
GB & $95,28 \%$ & $96,16 \%$ & $96,29 \%$ & $95,96 \%$ \\
XGBoost & $\mathbf{9 6 , 7 5 \%}$ & $\mathbf{9 7 , 2 8 \%}$ & $\mathbf{9 6 , 9 9 \%}$ & $99,11 \%$ \\
MLP & $90,45 \%$ & $91,73 \%$ & $90,97 \%$ & $99,14 \%$ \\
\hline
\end{tabular}

\section{CONCUSIONS}

In this research, we compared eight different algorithms on the Wisconsin Breast Cancer dataset to classify breast cancer. Using 1-fold and 5-fold cross-validation, we collected the performance metric to determine the best algorithm. The result showed that XGBoost has the best performance metric against other algorithms, and also has the highest AUC of $99,61 \%$. We conclude that XGBoost is the most accurate algorithm to classify breast cancer using the Wisconsin Breast Cancer
dataset.In the future, XGBoost can be compared with other algorithms that have not been tested in this experiment and canbe tested on different datasets as well.

\section{ACKNOWLEDGMENTS}

This work is partially supported by the IndonesiaEndowment Fund of Education (LPDP), Ministry of Finance, Indonesia. 


\section{REFERENCES}

Amrane, M. et al. 2018. Breast cancer classification using machine learning. Electric Electronics, Computer Science, Biomedical Engineering's Meeting, pp. 1-4.

Asri, H. et al. 2016. Using machine learning algorithms for breast cancer risk prediction and diagnosis. International Symposium of Frontiers in Ambient and Mobile Systems, pp. 1064-1069.

Bayrak, E. et al. 2019. Comparison of machine learning methods for breast cancer diagnosis. IEEE Scientific Meeting on Electrical-Electronics and Biomedical Engineering and Computer Science, pp. 4-6.

Center for Machine Learning and Intelligent Systems (UCI Machine Learning Repository). Breast Cancer Wisconsin (Diagnotic) Dataset. 1995. https://archive.ics.uci.edu/ml/datasets/Breast+Cancer+Wiscon sin+(Diagnostic) [6 November 2019]

Derisma, et al. 2018. Optimization of neural network with genetic algorithm for breast cancer classification. International Conference on Information Technology Systems and Innovation, pp: $398-403$.

Gayathri, B. and Sumathi, C. 2016. An automated technique using gaussian naïve bayes classifier to classify breast cancer. International Journal of Computer Applications, pp. 16-21.

Gbenga, D. et al. 2017. Performance comparison of machine learning techniques for breast cancer detection. Nova Journal of Engineering and Applied Science, pp. 1-8.

Kumar A. et al. Machine learning based approaches for cancer prediction: a survey. International Conference on Advanced Computing and Software Engineering, pp. 326 - 330.
Liu, B. et al. 2018. Comparison of machine learning classifier for breast cancer diagnosis based on feature selection. Proceeding of IEEE International Conference on Systems, Man, and Cybernetics, pp. 4399 - 4404.

Maity, G., and Das, S. 2017. Machine learning for improved diagnosis and prognosis in healthcare. IEEE Aerospace Conference, pp. 1-9.

Pham, B. et al. 2017. Hybrid integration of multilayer perceptron neural networks and machine learning ensembles for landslide susceptibility assessment at Himalayan area (India) using GIS. Catena, pp: $52-63$.

Punmiya, R. and Choe, S. 2019. Energy theft detection using gradient boosting theft detector with feature engineering-based preprocessing, pp: $2326-2329$.

Sharma, S. et al. 2018. Breast cancer detection using machine learning algorithms. International Conference on Computational Techniques, Electronics and Mechanical Systems, pp. $114-118$.

Suyanto. 2018. Machine learning tingkat dasar dan lanjut. Informatika, Bandung.

World Health Organization. 2019. Early diagnosis and screening for breast cancer. https://www.who.int/cancer/prevention/diagnosisscreening/breast-cancer/en/ [13 November 2019]

World Health Organization. 2019. Key fact about breast cancer. https://www.who.int/en/news-room/fact-sheets/detail/cancer [13 November 2019] 
THIS PAGE INTENTIONALLY LEFT BLANK 